\title{
Scarf Osteotomy without Osteosynthesis in the Treatment of Hallux Valgus
}

R. El Ghadraoui ${ }^{*}$ A. Bensassi, R. Maanouk, W. Boughzala, A.Madani, R. Jarbouh, M. El Idrissi, A. EL Ibarhimi, El Mrini A, O. Kada

Orthopedic Service, Centre hospitalier de Gonesse, Paris, France

DOI: $10.36347 /$ sasjs.2020.v06i10.008

| Received: 07.10.2020 | Accepted: 21.10.2020 | Published: 30.10.2020

*Corresponding author: EL GHADRAOUI Redouane

\section{Abstract}

Original Research Article

Hallux valgus (HV) has been around for over a century and a half, the osteoarticular deformity of the forefoot having most stimulated the imaginations of surgeons interested in this pathology and its treatment. Numerous techniques and surgical variations have been described over the years reaching around 150 therapeutic procedures [1]. Scarf osteotomy is a technique described by Burutaran [2] in 1976, taken up by Weil [3,4] and Borelli in 1990 and popularized in France by Barouk.

Keywords: Hallux valgus, SCARF, osteotomy.

Copyright $\odot 2020$ The Author(s): This is an open-access article distributed under the terms of the Creative Commons Attribution 4.0 International License (CC BY-NC 4.0) which permits unrestricted use, distribution, and reproduction in any medium for non-commercial use provided the original author and source are credited.

\section{INTRODUCTION}

The technique of SCARF osteotomy of the first metatarsal in the treatment of $\mathrm{HV}$ has undergone regular development over the past 20 years, which allowed to modify his mode of osteosynthesis. The fixation seemed essential in the first series of the authors. We present the results of a study including 45 patients (50 feet) operated for HV by Scarf osteotomy without osteosynthesis (according to the Maestro technique) in 2017 and 2018. This is a retrospective study in which we have studied and evaluated clinical and radiological results to demonstrate the reliability and reproducibility of the technique we use. The technical modifications made to the Classic Scarf by Maestro consist in making a more oblique proximal transverse osteotomy cut, replacing the distal screw with an osteosuture and the proximal screw by the entrapment of the bone wedge, resected on the proximal fragment.

\section{MATERIALS AND METHODS}

The objective of our work is to evaluate the clinical and radiological results of the technique used in our department for the treatment of hallux valgus, namely the Scarf osteotomy without osteosynthesis. We reviewed, retrospectively, 45 patients (50 feet) operated by three practitioners, in the orthopedic surgery department of the Gonesse hospital-Paris between January 2017 and December 2018. Our patients had an average age of 54 years, and the majority $82 \%$ consulted for pain and deformity of the metatarsophalangeal (MTP) of the big toe. All our patients underwent standard X-rays of the foot in front and side load with measurement of the angle of the hallux valgus between the 1st metatarsal and the 1st phalanx (M1-P1), the metatarsus varus between the 1st and 2nd phalanx (M1-M2) and distal metatarsal articular angle DMAA. The position of the sesamoid strap was also specified. The average follow-up is 13 months.

We used the surgical technique described by Maestro in 1999. In dorsal decubitusby linear medial approach centered on the head of M1, and a second dorsal approach of the first intermetatarsal space to release the sesamoido-metatarsal-phalangeal complex. After medial arthrotomy, sparing bone resection of the inner surface of the metatarsal head is performed.

The osteotomy is performed, as described by Barouk, along a longitudinal line and two transverse oblique lines, proximal and distal (Fig 01). Correction of the metatarsus varus is obtained by translating the proximal fragment to the medial and the distal fragment to the lateral (fig 02). 


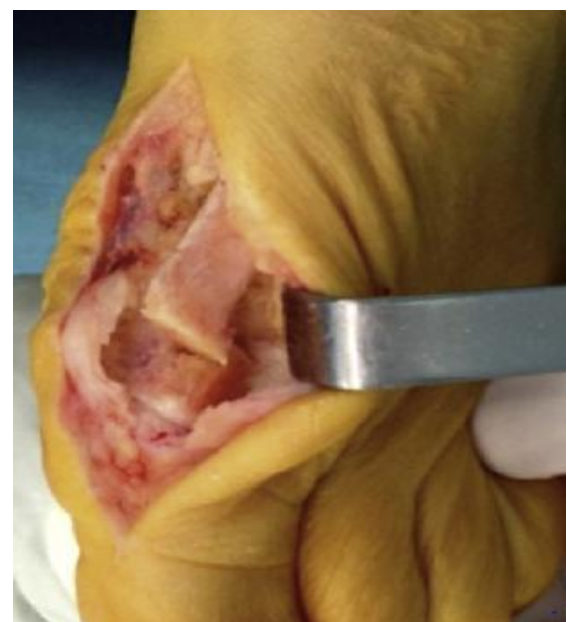

Fig-1: Making the transverse proximal cut line at $30{ }^{\circ}$ improves stability

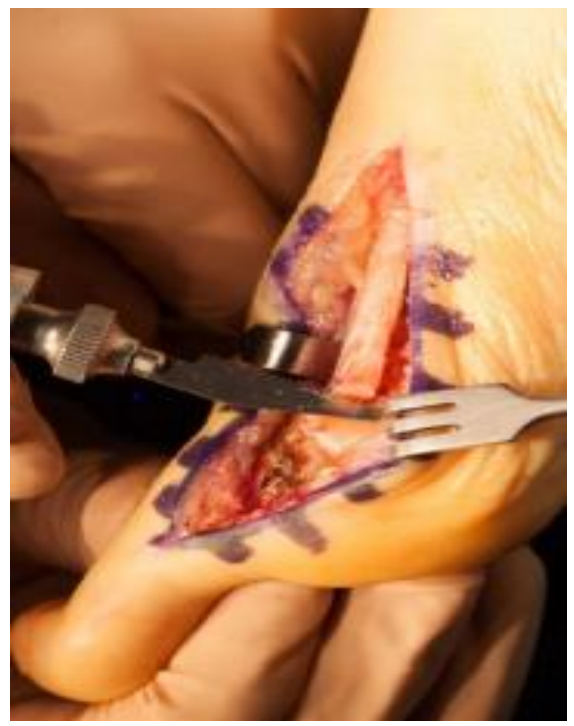

Fig-2: Translation of the lower and plantar part of the metatarsal

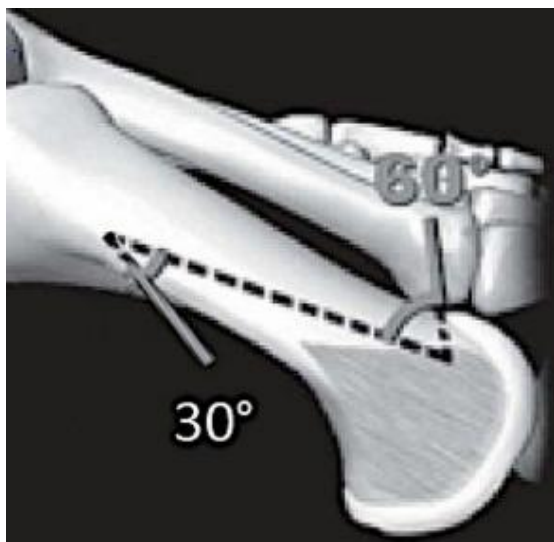

Fig-3: Resection of the protruding part

We resect the protruding part of the proximal fragment, which we embed in the proximal part of the osteotomy (Fig 03), which gives more stability and minimizes bone substance loss. We reinforce the stability of the assembly by an osteosuture on the metaphyseal region with a self-locking knot (Figs 04,05). All patients were hospitalized for two days and were put on preventive anticoagulation for three weeks. Support is authorized immediately after surgery with suitable heel support shoes for a period of one month. Passive and active mobilization with metatarsophalangeal support is prescribed for all patients and started immediately after the operation.

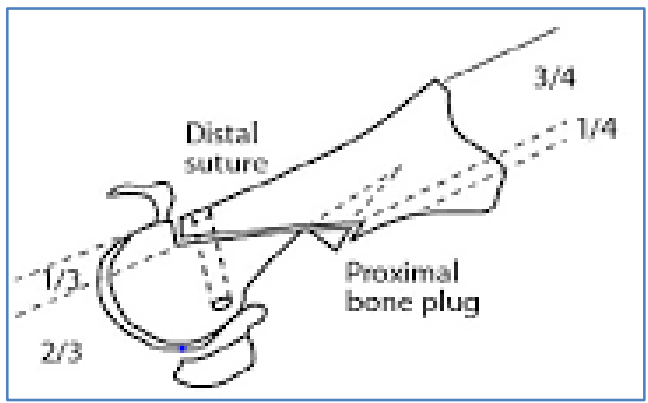

Fig-4: Description by Maestro osteosuture

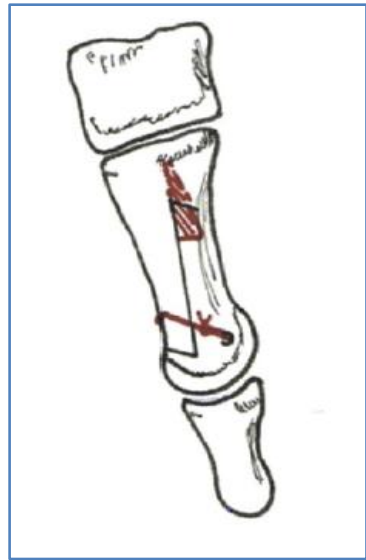

Fig-05: Intraoperative image

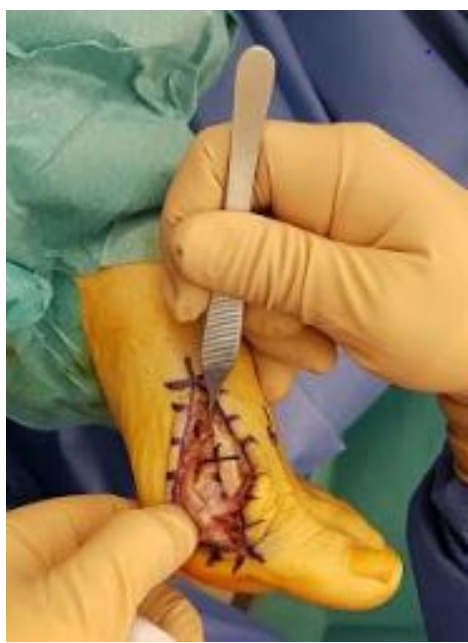

Fig-6: shematic medial of the view of the osteosuture

\section{RESULTS}

These are 37 women and 8 men, with an average age of 54.2 years (24-65 years). $80 \%$ presented with pain and deformation of the metetarsophalangeal joint of the big toe and $10 \%$ had associated bursitis. We used the Kitaoka functional score which presented a mean value of 53/100 preoperatively. The analysis of the X-rays showed an M1-P1 angle of $41.36^{\circ}, \mathrm{M} 1-\mathrm{M} 2$ 
$18.34^{\circ}$ and DMAA of $23.9^{\circ}$ preoperatively. Our patients were operated on by three different surgeons and received only a SARF without any other associated procedure such as osteotomy of the proximal phalanx. The intervention was bilateral, at the same operating time, in five patients $(4 \mathrm{~F} / 1 \mathrm{H})$, and in patients operated on only one side, the right side was operated on in ten patients.

The postoperative Kitaoka functional score presents an average value of 91/100 with extremes of 73 to $95 / 100$. Regarding the radiological results, at the last follow-up, the metatarsophalangeal deformity was corrected with an average M1-P1 angle of $12.6^{\circ}$ postoperatively, on the other hand an improvement in the average M1-M2 Angle, increased to $13,11^{\circ}$ at the last follow-up. The DMAA first metatarsal epiphyseal valgus rose to $8.21^{\circ}$ with extremes of 5 and 16 degrees.

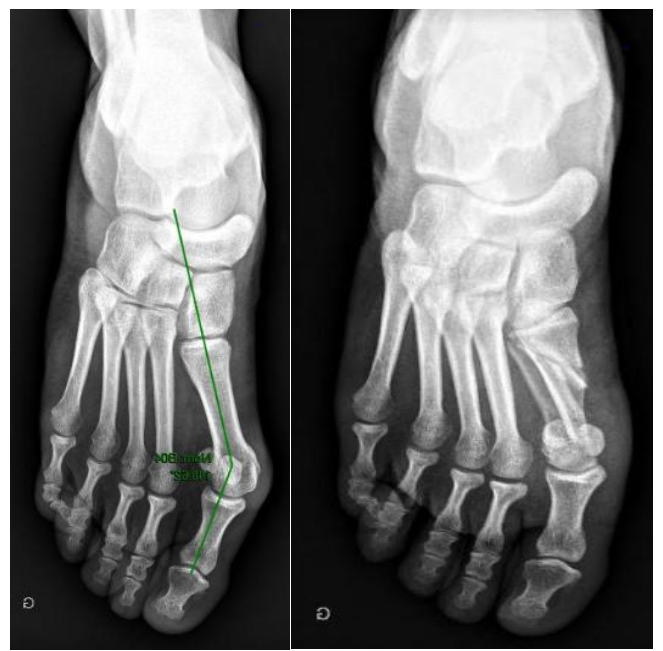

x-ray pre and post operative

The position of the sesamoid strap decreased from an average value of 3.7 to 1.6 at the last follow-up.
We recorded two cases of secondary displacement that did not require surgical revision, in addition we noted 01 cases of infection of the surgical site which progressed well under antibiotic therapy and local care. We noted no significant difference in clinical and radiological results between surgeons.

\section{DISCUSSION}

Modern foot surgery requires rapid functional recovery which cannot be achieved without the primary stability and strength of an osteotomy. A versatile and reliable technique adapting, thanks to the possibilities of three-dimensional correction, to moderate to severe hallux valgus, seemed essential tous. This development is motivated by the insufficiencies and complications found for chevron osteotomies, basal osteotomies and Lapidus-type arthrodesis [7].

The technical modifications made to the classic Scarf by Maestro consist in making a more oblique proximal transverse osteotomy line, and a notch at the level of the medial side of the metatarsal head where the distal lateral corner of the fragment is embedded. proximal [5], replacement of the distal screw by an osteosuture and replacement of the proximal screw by the entrapment of the bone wedge, resected at the expense of the proximal fragment after its lateral translation, at the level of the proximal part of the osteotomy.

Maestro [6] compared the results of three sets of patients: a set of 49 feet who had a Scarf with two screw fixation, 37 feet a Scarf with a single screw fixation and 33 feet a Scarf without fixation. The table illustrates the comparison of these three series. He concludes that the correction is better, for the parameters studied, in the Scarf series without screws:

\begin{tabular}{|c|c|c|c|c|c|}
\hline & year & number & M1 P1 & M1 M2 & DMAA \\
\hline 02 screws & $93-95$ & 49 & $12,67+/-7,22^{\circ}$ & $7,93+/-2,61^{\circ}$ & $13,5+/-4,5^{\circ}$ \\
\hline 01 screw & $96-98$ & 37 & $13,11+/-6,67^{\circ}$ & $7,44+/-2,51^{\circ}$ & $6,89+/-4,27^{\circ}$ \\
\hline without screw & 99 & 33 & $7,07+/-4,84^{\circ}$ & $6,51+/-2,36^{\circ}$ & $7+/-3,8^{\circ}$ \\
\hline our series & $2017-2018$ & 50 & $10,7^{\circ}+/-5.1^{\circ}$ & $7,1^{\circ}+/-2,2^{\circ}$ & $6,53^{\circ}+/-3,9^{\circ}$ \\
\hline
\end{tabular}

The technique of correction of hallux valgus by a Scarf osteotomy without osteosynthesis is a reliable technique, reproducible and allowing better corrections in the different planes with a low rate of complications.
The learning curve of this surgery remains long, the respect and application of the various technical devices remain essential for this economical, reliable and biological procedure. 


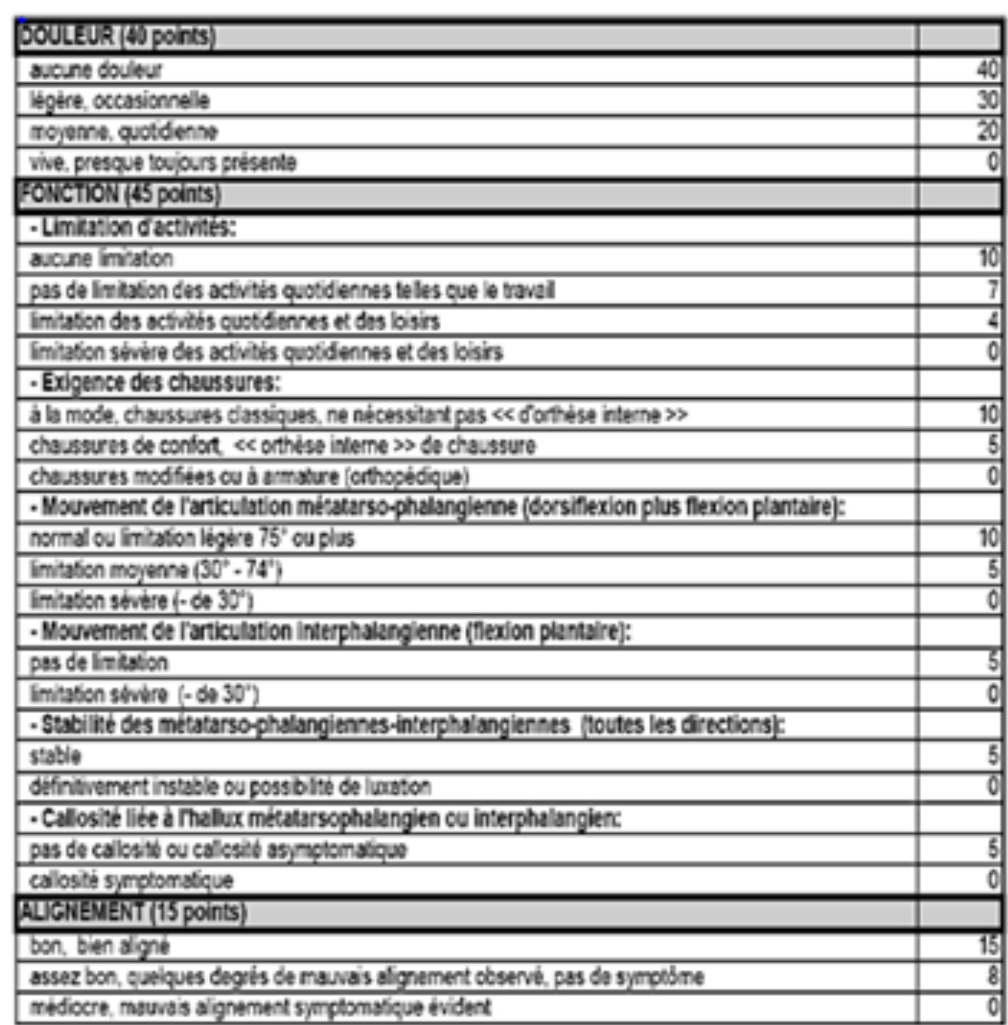

\section{Kitaoka functional score}

\section{CONCLUSION}

The scarf osteotomy without osteosynthesis meets all the conditions necessary to be a technique of choice in the treatment of hallux valgus, offering excellent results with a low rate of complications.

\section{REFERENCE}

1. Ferrari J, Higgins JP, Prior TD. Interventions for treating hallux valgus (abductovalgus) and bunions. Cochrane Database of Systematic Reviews. 2004(1).

2. Burutaran JM. Hallux valgus y cortedad anatomica del primer metatarsano (correction quinrusica). Actual Med Chir Pied. 1976;13:261-6.

3. Weil LS. Scarf osteotomy for correction of hallux valgus. Historical perspective, surgical technique, and results. Foot and ankle clinics. 2000 Sep;5(3):559-80.

4. Borrelli AH. Modified scarf bunionectomy: our experience in more than 1000 cases. J Foot Surg. 1991;30:609.

5. Valtin B. Hallux valgus: introduction. Pathologie du pied et de la cheville. Paris: Elsevier Masson ed. 2009:100-2.

6. Maestro M. Scarf osteotomy without screw fixation. Interactive Surgery. 2007 Jul 1;2(1):12-6.

7. Trnka HJ, Mühlbauer M, Zembsch A, Hungerford M, Ritschl P, Salzer M. Basal closing wedge osteotomy for correction of hallux valgus and metatarsus primus varus: 10-to 22-year follow-up. Foot \& ankle international. 1999 Mar;20(3):171- 